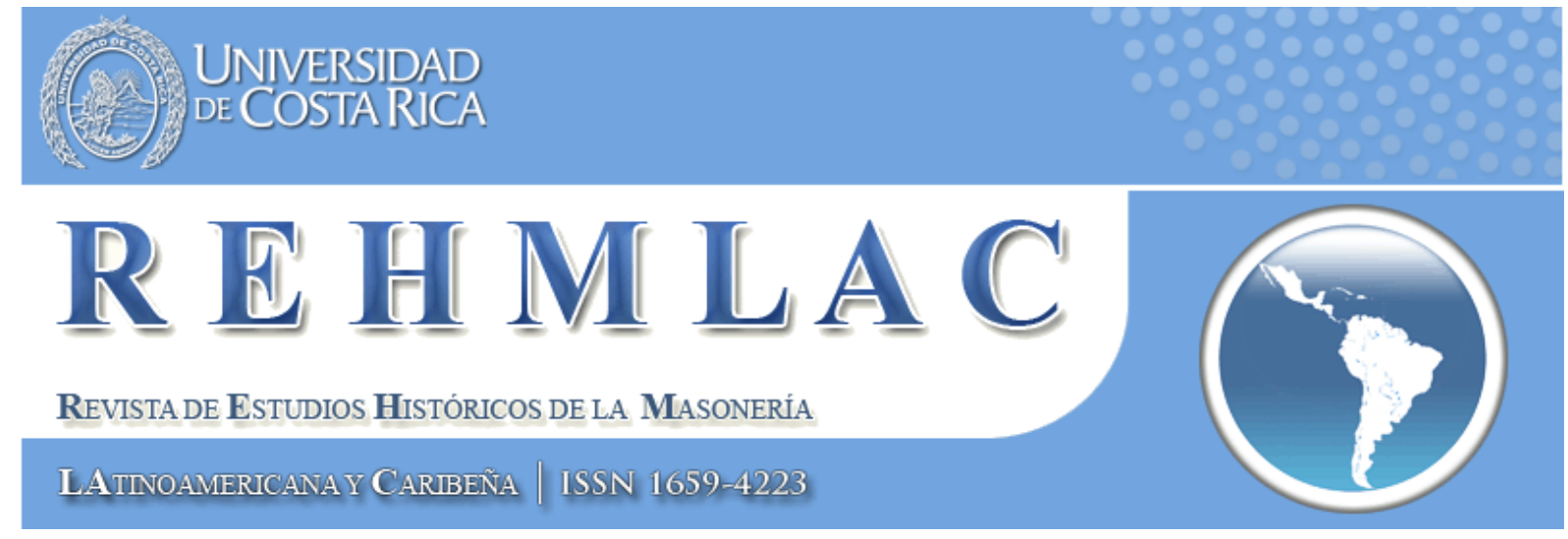

\title{
« La Maçonnerie française et la première guerre mondiale »
}

\author{
André Combes
}

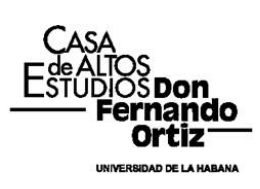


(C) André Combes et REHMLAC.

\section{Équipe REHMLAC}

Conseil scientifique :

Miguel Guzmán-Stein (Universidad de Costa Rica, Costa Rica)

José Antonio Ferrer Benimeli (Universidad de Zaragoza, España)

Margaret Jacob (University of California Los Angeles, United States)

Eduardo Torres Cuevas (Universidad de La Habana, Cuba)

María Eugenia Vázquez Semadeni (Stanford University, United States)

Eric Saunier (Université du Havre, France)

Andreas Önnerfors (Lunds universitet, Sverige)

Samuel Sánchez Gálvez (Universidad Carlos Rafael Rodríguez de Cienfuegos, Cuba)

Roberto Valdés Valle (Universidad Centroamericana “José Simeón Cañas”, El Salvador)

Céline Sala (Université de Perpignan, France)

Dominique Soucy (Université de Franche-Comté, France)

Guillermo de los Reyes Heredia (University of Houston, United States)

Dévrig Mollès (Université de Strasbourg, France)

Felipe Santiago del Solar Guajardo (Universidad ARCIS, Santiago de Chile)

Carlos Francisco Martínez Moreno (Universidad Nacional Autónoma de México, México)

Michel Goulart da Silva (Universidade do Estado de Santa Catarina, Brasil)

Examinateurs de style:

Sylvia Hottinger (Centro de Estudios Históricos de la Masonería Española, España)

Kyle Jackson (University of California Los Angeles, United States)

$W e b$ administrateur et designer:

Luis Martín Valverde Alfaro (Universidad de Costa Rica, Costa Rica)

Editor:

Yván Pozuelo Andrés (IES Universidad Laboral de Gijón, España)

E-mail: yvan@edu.xunta.es

Director:

Ricardo Martínez Esquivel (Universidad de Costa Rica, Costa Rica)

E-mail: ricardo.martinezesquivel@ucr.ac.cr

Adresse web : www.rehmlac.com/

E-mail : info@rehmlac.com

PO Box : 243-2300 San José, Costa Rica 
Cité dans :

Academia.edu

Aladin. WRLC. Libraries Catalog

AFEHC. Asociación para el Fomento de los Estudios Históricos en Centroamerica

Biblioteca de Georgetown

CRICCAL, Université Sorbonne Nouvelle Paris 3

CERGE EI. Portál elektronických časopisů. Univerzita Karlova v Praze

Departamento de Filosofía de la Universidad Centroamericana "José Simeón Cañas"

Dialnet, Universidad de la Rioja

Directorio y recolector de recursos digitales del Ministerio de Cultura de España

DOAJ. Directory of Open Access Journals

e-revist@s. Plataforma Open Access de Revistas Científicas Electrónicas

Freemasonry and Civil Society Program at UCLA

Fudan University Library Academic Resource Portal

Google académico

Institute for the Study of the Americas at University of London

Latindex (UNAM)

Latindex.ucr. Repositorio de revistas de la Universidad de Costa Rica

Library Catalogue of University of South Australia

Museo Virtual de la Historia de la Masonería de La UNED

Nuevo Mundo. Mundos Nuevos

REDIAL. Red Europea de Información y Documentación sobre America Latina

SciELO - Scientific Electronic Library Online

Sherpa/Romeo

SID. Sistema Integrado de Documentación. Universidad Nacional de Cuyo

Toronto Public Library

UBO. Revues en ligne. Université de Bretagne Occidentale

Universia. Biblioteca de Recursos

University of Saskatchewan Library

University of Wiscosin-Madison Libraries

Western Theological Seminary. Beardslee Library Journals 


\title{
« La Maçonnerie française et la première guerre mondiale »
}

\section{André Combes}

Directeur de l'IDERM (Institut d'Etudes et de Recherche maçonnique), rédacteur en chef de Chroniques d'Histoire maçonnique, membre du comité de rédaction d'Humanisme.

E-mail : andre.combes@neuf.fr

Date de réception: 20 août 2014 - Date d'acceptation: 15 octobre 2014

\author{
Mots-clé \\ Franc-maçonnerie, 1914, France, Première Guerre Mondiale.
}

Keywords

Freemasonry, 1914, France, World War I.

\section{Résumé}

La Première Guerre Mondiale rompit la fraternité universelle qui était la fierté de la Franc-maçonnerie. Que futil du pacifisme ? Comment la franc-maçonnerie française agit-elle face à ce conflit ? Quelles relations maintintelle avec le reste des obédiences étrangères?

\begin{abstract}
World War I fractured the universal brotherhood that Freemasonry had previously boasted. What was pacifism? How did French Freemasonry address the conflict? How was it related to other masonic obediences?
\end{abstract}

\section{Introduction}

Le Grand Orient de France (GODF) réunit 460 loges en 1914, avec des effectifs stables depuis 1908, d'environ 31.000 membres. Son président Georges Corneau, un radical, est directeur du journal Le Petit Ardennais. La Grande Loge de France (GLDF) compte 144 loges et 8.400 membres, sous le maillet de Gustave Mesureur, ancien ministre, directeur de l'assistance publique de la Seine. Le Droit Humain, qui traverse des heures difficiles est une petite obédience mais la seule à demander que le droit de vote soit immédiatement accordé aux femmes. Quelles relations établirent les obédiences françaises avec leurs homologues? Comment vécurent-elles ce conflit?

\section{Avant la guerre}

En 1913, année préélectorale, le GODF ne prend pas position sur le projet de loi portant, du fait des tensions internationales, le service militaire de deux à trois ans. Il adresse le 18 mars 1913, une circulaire dénonçant «les excitations chauvines d'où qu'elles viennent », mais aussi fait appel « au patriotisme et à la sagesse du Parlement» concluant cependant que le service de trois ans ne saurait être admis que s'il est absolument prouvé qu'il est indispensable. Bien que les élections législatives soient gagnées par la gauche, le retour aux deux ans est ajourné. Le nouveau Président du Conseil, René Viviani, républicainsocialiste est maçon, à la différence de ses prédécesseurs. 
Pendant les guerres des Balkans (1912-1913), le Conseil de l'Ordre reste neutre mais soutient divers organismes pacifistes : La Paix par le Droit, la Société d'arbitrage entre les Nations, la Ligue internationale de la Paix et de la Liberté, L'Union interparlementaire, à l'origine de la conférence de La Haye. Il adhère en décembre 1912 au Bureau international de la Paix siégeant à Berne créé en 1891 pour coordonner les actions des sociétés pacifistes.

Un des thèmes récurrents dans les exposés en loge de 1912 à Juillet 1914 est celui du rapprochement franco-allemand. La Maçonnerie germanique est très différente de la française. Elle comprend huit obédiences dont les Grands Maîtres adoptent des décisions communes au cours de rencontres périodiques. Les trois obédiences prussiennes qui regroupent 40.000 membres sont protestantes, monarchistes et nationalistes. A leur côté, les cinq obédiences « humanitaires » sont plus libérales, mais ne comptent que 18.000 adhérents. Le courant socialiste ne s'y est pas implanté.

Les relations avec le GODF avaient été rompues à la suite de l'accusation de crimes de guerres portées en 1870 contre le roi de Prusse et son fils, tous deux maçons, puis par l'annexion de l'Alsace-Lorraine et sa conséquence, la fermeture des loges du GODF. Les premiers rapprochements s'esquissent au cours de congrès maçonniques internationaux, d'abord avec la GLDF puis plus difficilement avec le GODF. Trois obédiences, sises à Hambourg, Bayreuth et Francfort, signent des traités d'amitié avec lui. Des manifestations internationales périodiques puis annuelles ont facilité ce rapprochement. Elles se tiennent à Luxembourg en 1912 puis à La Haye en 1913. Un rendez-vous est pris pour le 16 août 1914 à Francfort.

La question des relations diplomatiques entre les deux pays est renvoyée par le convent de 1913 au Conseil de l'Ordre Un rapport est présenté par André Lebey lors de sa séance du 6 avril 1913. Il souligne que par principe un franc-maçon veut la paix mais que le " chauvinisme accentué » progresse en Allemagne, note que des Alsaciens ne souhaitent pas un nouveau carnage, que le Reich n'a pas pu assimiler l'Alsace-Lorraine et qu'elle pourrait devenir la terre du rapprochement, un trait d'union entre les deux pays si l'Allemagne lui accordait l'autonomie par une constitution indépendante. Il souligne que l'alliance russe a été nouée dans un but pacifiste et qu'une entente de la France, de l'Angleterre et de l'Allemagne assurerait la paix du monde. Ce texte est soumis à l'étude des loges par le Conseil au cours de sa séance du $1^{\text {er }}$ juillet 1914 mais la menace de guerre se précise au cours de ce mois. Le GODF rend hommage le $1^{\mathrm{er}}$ août au citoyen Jaurès assassiné par un nationaliste et invite tous les maçons à assister à ses obsèques.

Le 2 août, le GODF et la GLDF envoient un télégramme commun au Gouvernement pour le remercier d'avoir tenté tout ce qui était compatible avec la dignité nationale dans l'intérêt de la paix mondiale. La Franc-maçonnerie française veut encore espérer que les efforts diplomatiques du Gouvernement de la République aboutiront à une solution pacifique ; mais quel que soit le résultat des négociations engagées, elle l'assure de son entier dévouement. 


\section{4-1916, à la recherche de l'alliance maçonnique}

Le 3 août, l'Allemagne déclare la guerre à la France. Le 4 août, le Conseil de l'Ordre adresse le télégramme suivant à René Viviani : «J'ai l'honneur de vous faire connaître que le Grand Orient de France, interprète fidèle des sentiments patriotiques de la Franc-maçonnerie française, vous renouvelle l'assurance de son entier dévouement au Gouvernement de la République. Comme il l'a fait en 1870, il met ses locaux à la disposition du Gouvernement ».

Le 7 août, alors que Liège résiste encore, il félicite par l'intermédiaire du Grand Orient de Belgique « la vaillante et héroïque Nation belge, dont la fière et courageuse attitude est saluée avec respect et enthousiasme par tous les Français ». Il fait savoir que des témoignages de sympathie lui sont parvenus d'Italie, du Portugal et de Roumanie. Par circulaire, il informe le 17 août les loges encore en activité que le Convent est annulé. A propos des évènements militaires et donc de la victoire de la Marne, il reprend les propos du généralissime (le nom de Joffre, franc-maçon en sommeil, n'est pas cité): «Le Gouvernement de la République peut être fier de l'armée qu'il a préparée ».

Le GODF demande à ses loges d'établir la liste de ses morts au champ d'Honneur, de ses blessés, de ceux qui se sont distingués, ont fait l'objet de promotions, ont obtenu des citations et de les transmettre à la fin des hostilités. Cette demande sera malheureusement sans suite et nous ne disposons pas de la liste des maçons morts en combattant ${ }^{1}$. Il participe à l'union sacrée en proclamant que «tous ceux qui ont répondu à l'appel de la patrie, quelles que soient leurs opinions politiques, philosophiques et sociales, sont de bons et excellents Français ayant droit au même respect et à la même admiration ». Il fait part de ses premières actions humanitaires ${ }^{2}$.

L'attitude de la Maçonnerie allemande est stigmatisée au cours de la séance du Conseil de l'Ordre du 13 décembre 1914 ainsi que celle du gouvernement du Reich. Il leur est en particulier reproché d'avoir voulu démontrer que la Triple Entente aurait été responsable de la guerre, d'avoir mis « au compte de l'héroïque peuple belge les actes de vandalisme, les cruautés et les atrocités que leurs propres nationaux ont commis en Belgique...et renouvelés en France ». Les Maçons allemands sont accusés d'avoir déshonoré " notre belle Institution », ce qui les met au ban de la Maçonnerie universelle. Georges Corneau se fait un plaisir de lire un extrait du journal maçonnique allemand Latomia du 7 novembre 1914 en réponse à un appel du Grand Maître du Grand Orient de Belgique, Charles Magnette à la Grande Loge de

\footnotetext{
${ }^{1}$ Parmi les morts au Champ d'Honneur figurent le député radical Félix Chautemps, le Conseiller d'Etat Collignon, ancien secrétaire général de la présidence de la République, Vénérable en 1913 de la Loge AlsaceLorraine, engagé au $46^{\text {ème }}$ régiment d'infanterie, soldat de $1^{\text {ère }}$ classe, tombé à l'âge de 58 ans, le 15 mars 1915 , Henri Durre, député socialiste et l'instituteur socialiste Platel, Conseillers fédéraux de la GLDF, Eugène Jacquet, de la Loge L'Etoile du Nord à Lille, dénoncé pour avoir aidé des militaires à franchir les lignes allemandes, fusillé ainsi que trois autres patriotes. Une loge à Buenos Aires choisira son nom pour titre distinctif.

${ }^{2}$ Un service médical de consultations gratuites qui a reçu au cours des trois premiers mois 7.000 personnes (et 80.000 jusqu'en 1918) est installé au siège rue Cadet, suppléant ainsi au manque de médecins et il participe à diverses œuvres dont le Sac du soldat fondé par le Frère Gratia, du Libre Examen. Des centres de soin sont fondés par des loges. Le plus important est l'hôpital de Toulouse. Des réfugiés des zones occupées sont hébergés par des Frères. La GLDF organise un service de ravitaillement aux prisonniers, ouvre, avec le Frère Nicol, une maison de convalescence pour les Frères malades ou blessés.
} 
Darmstadt l'invitant de former une commission de Maçons des pays neutres pour visiter les pays dévastés par la guerre. Le Grand Maître Suss lui écrit «qu'il a une confiance inébranlable en notre armée, et la conviction que dans la guerre scélérate déchaînée contre nous, ils agiront humainement et que les pays occupés seront maintenus humainement dans l'ordre ». D'où en réaction le vote d'un communiqué adressé à la presse et aux Puissances maçonniques étrangères dénonçant la Maçonnerie allemande, et l'envoi d'un exemplaire du Livre jaune, relatif aux négociations ayant précédé l'entrée en guerre.

Au cours des premiers mois de guerre, se met en place un cabinet d' " union sacrée " autour de Viviani, avec quatre maçons sur quatorze ministres. Il est ensuite élargi à deux socialistes SFIO : Jules Guesde et Marcel Sembat (ministre des Travaux Publics), Conseiller de l'Ordre du GODF'.

Après la signature le 26 avril 1915 du traité secret de Londres, l'Italie rejoint la Triple Entente le 23 mai, en dépit de l'hostilité des socialistes, du Vatican et de libéraux comme Giolitti, pour récupérer les «terres irrédentes » soit le Trentin, Trieste, la région julienne et une partie de la Dalmatie, bien que ces deux dernières régions soient largement peuplées de Serbes, de Slovènes ou de Croates. Une Légion italienne $\left(4^{\text {ème }}\right.$ régiment de marche du $1^{\text {er }}$ étranger) formée de volontaires dirigés par le Frère Lieutenant-colonel Peppino Garibaldi, s'est déjà engagée en décembre 1914 dans l'Argonne. Parmi les premiers morts au combat, des Maçons comme Bruno (tué le 26 décembre) et Costante (tué le 5 janvier) Garibaldi ainsi que Chiossone, le Vénérable de la Loge Italia. L'organe des obédiences allemandes, le Grosslogenbund, soutient que l'Italie est entrée en guerre à cause de l'influence de la francmaçonnerie italienne qui, inspirée par la française, a fait pression sur le gouvernement Salandra. Cette accusation sera popularisée, après la guerre, par le ministre allemand Erzberger. Cette affirmation, bien que reprise par Raqueni Garibaldi, secrétaire général de Ligue franco-italienne qui devait la juger valorisante, est sans fondement. Si les relations entre le GODF et le GOI sont amicales, si les deux Maçonneries partagent en commun la défiance à l'égard du Vatican et une aspiration commune pour la démocratie et la laïcité, les relations entre elles sont purement épistolaires et, en outre, aucune des deux ne dispose de l'influence qu'on a pu leur prêter ${ }^{4}$.

Il en est de même pour d'autres pays, où les maçons, démocrates et parfois socialistes, sont favorables à l'Entente par sympathie pour la Révolution française et hostilité à l'égard des puissances centrales autocratiques. Mais, à l'exception du Portugal dont le Président de la

\footnotetext{
${ }^{3}$ Les ordres du jour des loges parisiennes tranchent singulièrement avec ceux des mois précédents : «L'armée et la République », « La dernière des guerres », «Les orphelins de la guerre », «Est-on à la veille de l'intervention italienne ? », «Le socialisme et la guerre », « De la liberté de conscience dans les hôpitaux militaires » et « Des femmes, des jeunes filles violées par les Allemands sont enceintes de leurs œuvres. Que faut-il faire ?».

${ }^{4}$ Le Conseil de l'Ordre du GODF adresse le 26 avril au GO d'Italie (GOI) un «salut affectueux» ajoutant : « Luttant côte-à-côte contre la barbarie et pour le triomphe du Droit et de la Civilisation, la France et l'Italie vont affirmer de nouveau, sur les champs de bataille et dans la victoire, leur union à jamais indissoluble ». Le GOI remercie le 23 juin alors que l'Italie « forte de son droit, prend les armes pour le triomphe du principe des Nationalités et pour la grande cause de la Civilisation et de la Justice ». Il n'eût eut pas de rencontres entre les dirigeants des obédiences avant les conférences de 1917 puis au lendemain de la victoire quand, sous la présidence du GM du GODF Georges Corneau, s'ouvre en loge un débat sur la politique économique et financière des deux pays en présence de Maçons italiens, dont le ministre d'Etat Barzilaï, et le secrétaire d'Etat à l'aviation Chiesa.
} 
République Bernardino Machado est maçon, ils n'exercent aucune influence. L'Allemagne déclare la guerre à ce pays le 9 mars 1916, c'est à la suite de la saisie, à la demande de l'Angleterre, de navires marchands allemands réfugiés dans ses ports; mais pour le gouvernement $\mathrm{du}$ Portugal ce conflit répond aussi à la nécessité de protéger les colonies africaines de la rapacité allemande et de légitimer la jeune République. Selon le GODF cependant, le Grand Orient Lusitanien Uni (GOLU) aurait joué un rôle dans cette décision et son Grand Maître, Magalaès Lima fera en mai 1916 une tournée triomphale dans des loges françaises à Paris, Toulouse, Montpellier, Bordeaux. Le GOLU, dans une correspondance avec le GODF, précise que «la race latine qui a toujours lutté pour la liberté ne pouvait manquer de s'unifier pour combattre l'ennemi commun : les barbares du Nord ». A l'opposé, le Grosslogenbund à l'issue de sa $40^{\text {ème }}$ Diète envoie un télégramme au Kaiser attaquant les Maçonneries des pays engagés contre l'Allemagne (bien que celles de France et britanniques soient opposées) traitées d' " associations secrètes dégénérées ».

La GL suisse Alpina qui dispose du Bureau International des Relations Maçonniques établit des questionnaires pour pouvoir transmettre des nouvelles des prisonniers civils ou militaires. Elle ouvre en 1915 un Bureau pour la recherche des disparus. La Loge belge Albert de Belgique lance un appel aux Maçons US pour qu'ils n'appuient qu'un projet de paix incluant l'évacuation de leur territoire.

Le Conseil de l'ordre, dans sa séance du 17 mai 1916 invite les loges à étudier les conditions de l'Europe nouvelle au lendemain de la guerre. : l'organisation politique et sociale de la France, les rapports du capital et du travail, les droits de la femme dans le travail, alors qu'elles sont employées dans les usines de guerre, mais en tenant compte que « la véritable place de la femme est au foyer », la reconstruction des zones occupées et comment s'affranchir de la vassalité économique imposée par l'Allemagne. Le comité des loges parisiennes en novembre 1916, au cours d'une Tenue en présence de maçons anglais, italiens et suisses, adresse son salut à la nation serbe ; puis en décembre, organise une Tenue au grand Temple de la rue Cadet en présence des Maçons belges, anglais (donc sans l'accord de leur obédience), russes, italiens et serbes.

\section{7 , le tournant}

En 1917 le GODF et la GLDF organisent une réunion des Maçonneries des nations alliés pour élaborer un programme d'action commune pour permettre à la Franc-maçonnerie de faire entendre «sa grande voie humanitaire ». Elle se réunit rue Puteaux, au siège de la GLDF, les 14 et 15 janvier. Les quatre obédiences françaises représentées sont le SCDF et le GCDR (qui gèrent les hauts grades), les GODF et la GLDF. Les obédiences étrangères sont le GO Lusitanien Uni (Magalaès Lima), le SC et le GO d'Italie (Ettore Ferrari ${ }^{5}$ pour le GODI), le SC et le GO de Belgique (Anspach et Jennissen), le SC de Serbie (Yovanovich et le colonel Ilich). Six résolutions sont adoptées. La première pour dénoncer les mesures de déportation et

\footnotetext{
${ }^{5}$ Ettore Ferrari croyait qu'il s'agissait d'un congrès entre socialistes et il écrit en septembre 1916 au GODF pour rappeler que le Parti socialiste officiel est contre la guerre à la différence du parti socialiste réformiste qui doit être seul invité.
} 
de travail forcé, notamment celles de jeunes garçons enlevés en Serbie pour les germaniser, la seconde, pour condamner les tueries et les cruautés commises par l'armée turque avec la complicité des officiers allemands en Arménie, en Syrie et au Liban, les deux suivantes pour remercier les Maçons des Etats-Unis et de Suisse pour leur appui, la cinquième est un hommage à l'ancien Grand Maître du GO de Belgique Charles Magnette, condamné et déporté pour avoir dénoncé celle de compatriotes, la sixième pour s'indigner du torpillage des bateaux de commerce, des incendies et pillages des villes et du survol des cités par les zeppelins afin de provoquer la terreur. Il est prévu qu'une nouvelle réunion sera élargie aux puissances maçonniques des pays neutres. L'appel souligne que la Maçonnerie est la seule Institution à avoir maintenu un caractère universel, que la guerre actuelle est une guerre pour la paix et la sécurité des petites nationalités et contre le militarisme et que la victoire des alliés sera celle du pacifisme... Au cours de la Tenue de clôture devant 1500 maçons, Charles Richet, futur prix Nobel de médecine, prononce un discours en faveur de la Société des Nations.

Le second congrès se tint du 28 au 30 juin 1917. Le GO d'Italie est représenté par les Frères Nathan, Ferrari et Berlanda (officieusement chargé de rendre plus fraternel les relations entre la Loge italienne de Tunis et les loges françaises) et la GL symbolique d'Italie par Meoni, son Grand Orateur. Les pays neutres présents sont le Grand Orient espagnol et la Grande Loge régulière Catalano Balear, tous deux très francophiles, la GL suisse Alpina et le SC de Suisse ainsi que le GO et le SC d'Argentine. D'autres obédiences sont excusées comme les GL de l'Ohio ou de l'Arkansas.

André Lebey, dans son allocution, lors de la première séance, situe ce congrès dans la continuité des conférences de la paix de 1899 à La Haye et de 1907. Il souligne qu'il n'y aura aucune possibilité de paix tant que le militarisme germanique restera debout, qu'il faudra mettre le peuple allemand dans l'impossibilité d'être asservi par ses anciens maîtres et que le pays soit régénéré afin de pouvoir entrer à la SDN. Les quatre conditions de la paix sont la restitution de l'Alsace-Lorraine, la reconstitution de la Pologne divisée en trois tronçons, l'indépendance de la Bohème et la libération ou l'unification «de toutes les nationalités aujourd'hui opprimées par l'organisation politique et administrative des Habsbourg en des états que les dites nationalités expriment par un plébiscite ». En note de bas de page, il est précisé que Trentin et Trieste reviennent de droit à l'Italie. Le Luxembourg restera neutre et la Belgique deviendra un terrain de rapports internationaux pacifiques.

Le général Peigné, Grand Maître de la GLDF, préside la seconde séance où on débat de la future SDN. Lebey apporte les conclusions comprenant 13 points. Les premiers posent les principes. Ainsi, le $3^{\text {ème }}$ définit le fondement de la nation comme la souveraineté manifestée par la volonté librement exprimée des populations, le $4{ }^{\text {ème }}$ proclame l'inviolabilité de l'indépendance des nations. Puis Lebey esquisse un projet pour la SDN. ${ }^{6}$

\footnotetext{
${ }^{6}$ La représentation internationale formera son Tribunal suprême. Le pouvoir législatif sera aux mains d'un Parlement comprenant 7 représentants par Etat. Il pourra s'adjoindre des commissions appropriées. Le Conseil des Nations exercera le pouvoir exécutif. Il comprendra un seul membre par nation, choisi par le Parlement international en son sein. Une Cour internationale de justice dont les membres seront élus pour trois ans représentera le pouvoir judiciaire. La guerre sera interdite. Le pays responsable sera sanctionné par des peines
} 
Peu d'amendements sont votés au cours des débats. Le congrès, suivant Meoni, « apporte au peuple le droit de reconstituer toutes les nationalités brisées ou opprimées en tenant compte de tous les éléments qui composent une conscience nationale ». Deux adresses aux Frères le Président Wilson et Léon Bourgeois sont adoptées. De retour en Italie, le GrandMaître Ferrari sera accusé de ne pas avoir précisé les noms de tous les territoires réclamés par l'Italie et devra abandonner sa charge.

Face à l'impossibilité de réunir un convent, le Conseil de l'Ordre décide d'organiser une réunion des vénérables ou délégués qui se tient du 20 au 22 septembre 1917. Il doit traiter de la défense du régime républicain, de la laïcité, de la forme démocratique de la nation et de la révision de la Constitution, de la réorganisation et à la reconstruction des provinces envahies, de la lutte contre l'alcoolisme, de la paix par la SDN. Selon les conclusions, les charges résultant de la guerre devraient être financées par un impôt progressif basé sur la quotité des revenus de chacun et les sommes drainées par les banques utilisées pour les grands travaux nécessaires à la reconstruction.

Les délégués décident que le convent se réunira en 1918 pour traiter, outre les questions maçonniques prévues pour celui de 1914, de la défense républicaine, de l'enseignement, de la réorganisation économique de la France, des colonies et des pays du protectorat. Il sera, du fait de l'offensive allemande, reporté du $1^{\mathrm{er}}$ avril au 16 septembre 1918. La victoire est alors certaine et le convent fait le point sur la guerre et s'intéresse surtout aux conséquences économiques et politiques prévisibles.

Les deux évènements majeurs au cours de l'année 1917 sont les deux révolutions russes et l'entrée en guerre des Etats-Unis. Celle de février suscite l'enthousiasme à la différence de celle d'octobre qui met un terme à l'alliance russe. Le GODF rappelle son attachement à l'union sacrée, situant (pour contrer la propagande pacifiste en son sein ?) son action dans la continuité de 1789 et dans celle de 1870, s'appuyant sur Blanqui, pour prétendre que patrie, république, esprit révolutionnaire et socialisme sont indissolublement liés. Il est bien représenté au sein du gouvernement Clemenceau formé en décembre 1917 avec Stéphan Pichon aux Affaires Etrangères (mais il ne jouera qu'un rôle mineur dans les négociations pour les traités de paix), Klotz et Louis Lafferre ancien président du GODF, ministre de l'Instruction Publique et des Beaux-Arts. En cette année, le socialiste Arthur Groussier, futur président du GODF, est vice-président de la Chambre des députés. Le courant léniniste au sein de la Maçonnerie ne s'affirmera qu'ultérieurement.

L'entrée en guerre des Etats-Unis le 6 avril 1917 suscite d'autant plus l'enthousiasme des Maçons français que le président Wilson est démocrate et Frère. Dès leur arrivée en France, des militaires américains sont invités par des loges et il va en résulter l'établissement de relations officielles entre quelques Grandes Loges américaines et l'une ou les deux obédiences françaises.

Les travaux des loges de 1916 à 1918 reflètent l'évolution du conflit. Certaines Tenues sont informatives comme en avril 1916 sur les relations entre l'armée et le haut commandement présenté par Accambray, député radical de l'Aisne. Certaines sont sociales

allant du boycott à l'isolement absolu. Le Parlement procèdera à une limitation des armements et fixera le lieu de ses réunions. La ville choisie sera la capitale du monde. 
telle la question des loyers et de la vie chère, par l'anarchiste Sébastien Faure ; d'autres, plus polémiques, comme la recherche des causes et des responsabilités des premiers revers par le Frère X, militaire, les débats sur la paix sans annexions ni indemnités (avril 1917), ou sur le livre « Le Feu » d'Henri Barbusse. Le 31 mai, un exposé sur « le droit de grâce et la justice militaire » pourrait être en rapport avec les mutineries. En décembre 1917, le député radical Hubbard traite de la fin de la guerre par la constitution mondiale alors que le socialiste Rozières, favorable aux Bolcheviks, se félicite d'un vent qui souffle en Russie. En 1918, sont évoqués l'affaire Malvy ${ }^{7}$ (défendu par les maçons dont Marcel Sembat lors du convent), la situation des régions envahies puis, après le 11 novembre 1918, les loges tirent des batteries de deuil pour leurs morts et d'allégresse pour le retour de l'Alsace-lorraine dans la mère patrie. Parmi les sujets abordés après l'armistice : la question des mutilés de guerre, le cas du Frère le capitaine Sadoul devenu léniniste, celle des officiers cléricaux et du clergé, notamment des aumôniers militaires, pendant le conflit.

$* * *$

La Première Guerre Mondiale secoua les principes universels de la maçonnerie. Les divisions maçonniques suivirent les divisions nationales opérées pendant le conflit. Après, s'en est suivi un long processus de rapprochement entre celles-ci freiné par leur principal ennemi : le contexte politique.

\section{Bibliographie}

Chevallier, Pierre. Histoire de la franc-maçonnerie française. La Maçonnerie : Église de la République (1877-1944), Tome 3. Paris : Fayard, 1975.

Combes, André. Les trois siècles de la Franc-maçonnerie française. Paris : Dervy, 2006.

Combes, André. Le grand-Orient de France 1865-1914. Paris: Edimaf encyclopédie maçonnique, 2009.

Ligou, Daniel. Histoire des Francs-Maçons en France. Paris : Editions Privat, 2000.

\footnotetext{
${ }^{7}$ Ancien ministre de l'Intérieur, radical, pacifiste, faussement accusé de trahison.
} 